\title{
International Journal of Sciences
}

\section{Study on the Effect of High Expression of Linker for activated T Cells in Nude Mice Tumor Model}

\section{ZHANG Min-Rui', GAO Mei-Hua1 ${ }^{1}$, MA Yu ${ }^{1}$, WANG Bing ${ }^{1}$, ZHANG}

\section{Bei ${ }^{1}$}

${ }^{1}$ Department of Immunology, Medical College of QingDao University, Qingdao 266000, Shandong Province, China

\begin{abstract}
BACKGROUND: The activation and proliferation of $\mathrm{T}$ cells are based on the signal transduction after combinating of ligands and T cell antigen receptors(TCRs). As an adapter molecular, LAT(Linker for Activation of T Cells) is a kind of transmembrane protein which leads to a positive regulation for growth and differentiation of $\mathrm{T}$ cells ${ }^{[1,2]}$. Then, the palmitoylation of LAT is the foundation of signal transduction process. OBJECTIVE: By construct an tumor model with $\mathrm{T}$ leukemia Jurkat cells in nude mice, to research the effect of high expression of LAT to tumor load of mice. METHODS: Culturing the Jurkat cell line, the 5 weeks old female node mice were classified to three groups: high expression of LAT group, LAT mutation group and normal Jurkat group, each group had 8 mice. After pretreated with cyclophosphamide, $5 \times 10^{6} /$ mice Jurkat cells were injected at armpit. Then observed the tumor of mice, measured weight of tumors, and HE stained of tumor tissues. Flow cytometry was used to detect proliferation of tumor cell in peripheral blood. ELISA was used to test the level of interleukia (IL)-2 in serum. RESULTS: After injected cells several days, the nude mice have appeared tumors under armpit and some symptoms like somnolent, anorectic and ect. The results of Flow cytometry and ELISA had shown that high expression of LAT group had the highest rate of tumor cells proliferation than other groups. CONCLUSION: It was successfully established the nude tumor model of T lymphocyte, confirmed the positive effect of LAT to the T cells activation and proliferation, provided a new target for acute T lymphocytic leukemia gene therapy.
\end{abstract}

Keywords: LAT, Nude, Tumor Model, Leukemia

\section{INTRODUCTION}

Balb/c nude mice (Balb/c-nude) is a new variety of mutant mouse formed in homozygosis of alleles of nude locus in VII linkage group. It has no thymus and cannot generate normal $\mathrm{T}$ cells. It has been reported that they had established animal model in Balb/c nude mice by tumor tissue xenograft ${ }^{[3]}$. Therefore, it is an ideal experimental animal model to study $\mathrm{T}$ lymphocytic leukemia.
LAT is a single-stranded transmembrane protein with the relative molecular weight of $36-38 \mathrm{KD}$ which mainly shows on $\mathrm{T}$ cells, NK cells,mast cells and platelets $^{[4-7]}$. The whole single strand is composed of an extracellular domain (containing 4 amino acids), a transmembrane domain (containing 22 amino acids) and a cytoplasmic domain (containing 208 amino acids). LAT is an important adaptor protein in $\mathrm{T}$

This article is published under the terms of the Creative Commons Attribution License 4.0

Author(s) retain the copyright of this article. Publication rights with Alkhaer Publications.

Published at: http://www.ijsciences.com/pub/issue/2015-09/

DOI: 10.18483/ijSci.821; Online ISSN: 2305-3925; Print ISSN: 2410-4477 
lymphocyte activation. When $\mathrm{T}$ cell is activated, the two cysteine residues on inner side of LAT membrane (Cys26 and Cys29) will undergo palmitic acidification $^{[8]}$. Thus, LAT will be located on glycolipid enriched microdomains (GEMs) on cell membrane. Then, it will make signal transduction together with other signal proteins ${ }^{[9]}$. If this site mutation occurs, it will prevent the combination of proteins and lipid rafts ${ }^{[10]}$. Therefore, this in vivo experiment aims to study the influence of LAT on T lymphocyte signal transduction through tumorigenesis in nude mouse.

\section{MATERIALS AND METHODS}

\section{Materials}

Cells

Juakat cells were saved in the Department of Immunology,Medical College of QingDao University.

\section{Animals}

Balb/c nude mice were purchased from Beijing Vital River Laboratory Animal Technology Co., Ltd. the certification No. was SCXK (Jing) 2012-0001. The mice were all female and 5 weeks old.

\begin{tabular}{|l|l|}
\hline Main reagents and instruments & \multicolumn{1}{l}{ Source } \\
\hline PRMI-1640culture, fetal bovine serum & Hyclone, USA \\
\hline Mouse-anti-human CD59 monoclonal antibody & BD Biosciences Company, USA \\
\hline cyclophosphamide (CTX, 0.2g/dose) & Jiangsu Hengrui Medicine ,China \\
\hline Fluoview FV1000 laser confocal microscope & Olympus, Japan \\
\hline Human IL-2 Elisa kit & USA
\end{tabular}

\section{Methods}

\section{Cell culture and transfections}

We used Juakat cell strain of human which is an acute leukemia T lymphocytic series. At first, transfected Juakat cells with EGFP (enhanced green fluorescence protein) lentivirus as the carrier. Thus, cell strains of LAT (LAT molecule high expression) and LAT-M (LAT palmitoylation site mutant) will be created. Then, used 100U/ml penicillin, 100U/ml streptomycin, $10 \%$ inactivated newborn calf serum and RPMI-1640 medium to prepare sterile complete medium. Cultured in the incubator under the condition of $37^{\circ} \mathrm{C}, 5 \% \mathrm{CO}_{2}$ saturated humidity.

\section{Animal}

Balb/c nude mice were fed in SPF laboratory. Strict aseptic operation was made. All articles used were all autoclaved before have been used.

\section{Tumorigenesis model}

Adjusted concentration of CTX to $20 \mathrm{mg} / \mathrm{ml}$. Gave intraperitoneal injection $2 \mathrm{mg}$ for each mouse for two days successively. On the third day, taken logarithmic phase Jurkat cell strains. Centrifuged and suspend. Gave subcutaneous injection $5 \times 10^{6} / 0.1 \mathrm{ml}$ LAT high expression cells, LAT palmitoylation locus mutation cells and normal Jurkat cells at armpits of different groups of mice respectively for two days in succession. Observed tumor formation situation of the mice after injection.

\section{Tumor tissue hematoxylin-eosin (HE) staining}

Taken tumor of the mice. Rinsed it with PBS solution, and then dipped it in $10 \%$ neutral formalin solution for fixation. After 48-72h, dehydrated, waxdipped, embedded and made it into paraffin section. After HE staining, observed tumor cell proliferation with microscope.

\section{Flow cytometry (FCM) detection}

Taken peripheral blood (PB) of the mouse. Added Mouse anti-human CD59 monoclonal antibody with PE mark. Incubated in dark for 20min. added red blood cell lysate and kept in dark for $10 \mathrm{~min}$. Washed 
twice with PBS. Precipitated and added PBS. Blended completely and sampled.

\section{ELISA testing}

Taken PB of the mouse. Centrifuged and collected the supernatant. Set 10 standard pores on enzyme label-coated plate. Added $50 \mu \mathrm{L}$ standard substance in different concentrations respectively. Added $10 \mu \mathrm{L}$ sample to be tested in to the sample pore. Added nothing to blank control pore. Set 4 duplicate pores for each pore. After sealed the plate with microplate sealers, made $37^{\circ} \mathrm{C}$ warm bath for $30 \mathrm{~min}$. Washed five times. Added $50 \mu \mathrm{L}$ enzyme label reagent in each pore except for blank pore. Made warm bath and clean. Added color developing agent. Developed in dark for $15 \mathrm{~min}$ in $37^{\circ} \mathrm{C}$. Stopped reaction. Tested absorbance (A) value at $450 \mathrm{~nm}$ wave length of each pore within $15 \mathrm{~min}$.

\section{Main outcome measures}

The weight and HE staining of tumors; Proliferation of cancer cells; The expression level of IL-2 in serum.

\section{Statistical analysis}

The paired-samples test was conducted for statistical analysis. Express the measurement data in the way of mean \pm standard deviation $\left(\bar{x}_{ \pm \mathrm{s}}\right) . P<0.05$ was considered significant. SPSS19.0 statistics software was used for analyze the data.

\section{RESULTS}

\section{Cells}

Under laser confocal microscope, As shown in Figure $1 \mathrm{~A}$, there was no green fluorescence in normal Jurkat cells. In Figure 1B-C, it shown green fluorescence, And in Figure 1B, we could saw green fluorescence focused on the cell membrane which had a bit gathering area, suggesting it may be lipid rafts. In Figure C, Jurkat cells transfected with LAT-M-EGFP, green fluorescence was in distribution, and couldn't saw the gathering area(Figure 1).
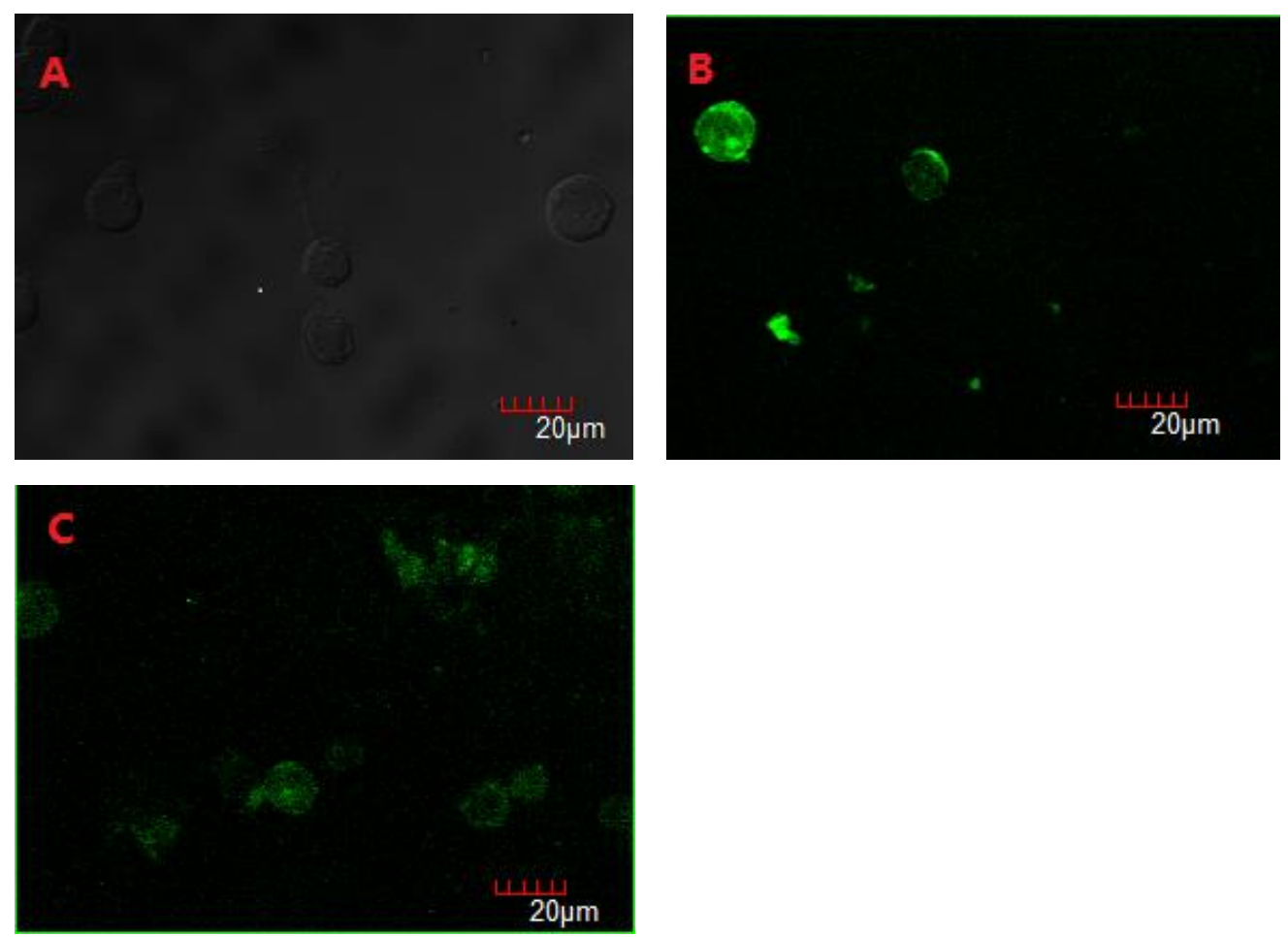

Figure 1 The green fluorescence under confocal microscope

Note: (A) Jurkat cells with no luminescence; (B) Jurkat cells transfected with LAT-EGFP; (C) Jurkat cells transfected with LAT-M-EGFP. All cells were stimulated by $488 \mathrm{~nm}$ luminescence. 


\section{Tumorigenesis of mice}

After been injected with tumor cells, the mice became somnolent and anorectic. There were visible lumps in subcutaneous tissue at armpits. With the lump gotten large, the weight reduced. Other symptoms included poor appetite and low spirit. Killed the mice in the fourth week. Taken out the

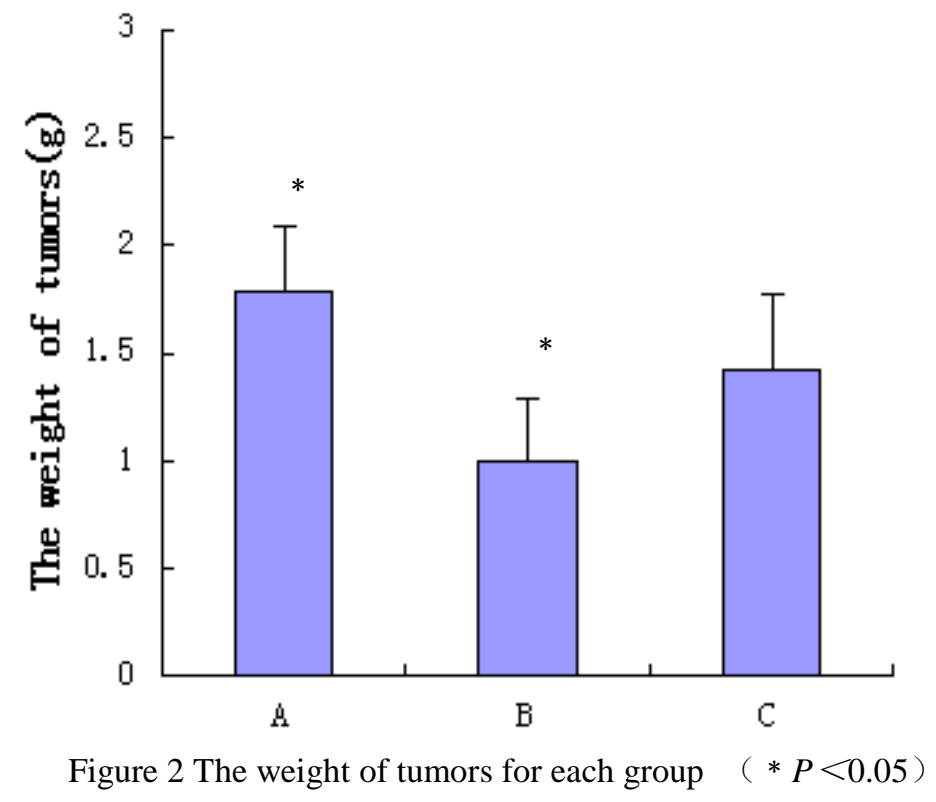

\section{HE staining of the tumor tissue}

Killed the mice and Taken out the intact tumor. The tumor had visible clear boundaries. The margin was irregular. After staining, you could saw tumor cells arranged in blade or gathered into cluster from the microscope. Boundaries of most cells were unclear. Compared with culture in vitro, the form had changed tumor tissue and weighed. Weight of the tumors of LAT high-expression group was higher than that of other two groups. That of LAT mutation group was lower than that of normal Jurkat cell group. By these comparison, $\mathrm{P}<0.05$. Thus, the difference had statistical significance (Figure 2).
A: LAT high expression group

B: Normal Jurkat group

C: LAT mutation group
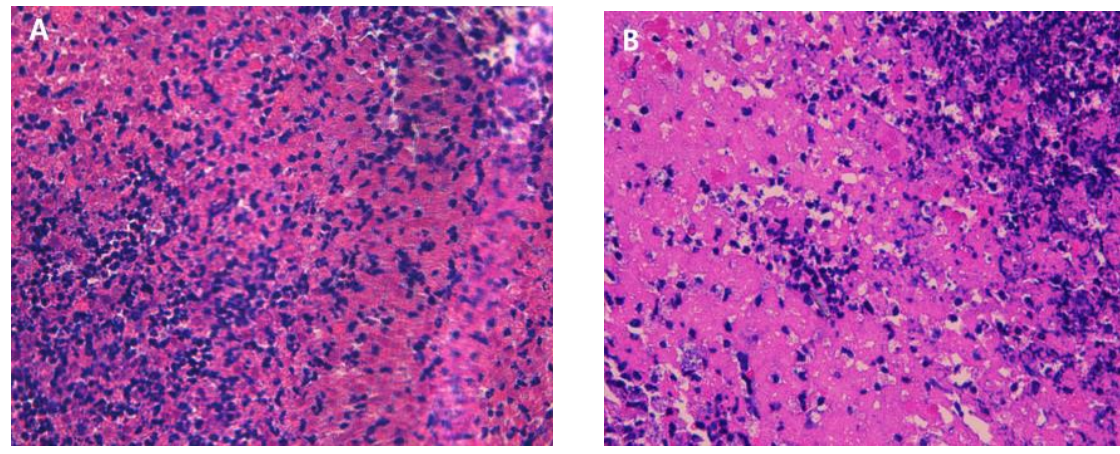

a lot. Cells had different sizes. Cell chromatins gathered in sheet. Compared with LAT mutation group and normal Jurkat group, tumor cell aggregation of LAT high expression group was more obvious, and cell proliferation was more violent. It showed that LAT promoted $\mathrm{T}$ lymphocyte proliferation (Figure 3). 


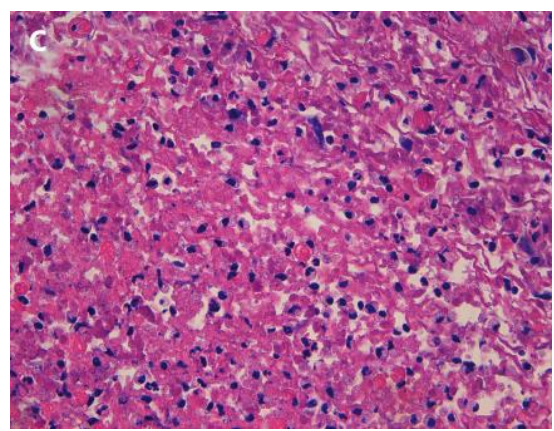
A: LAT high expression group
B: Normal Jurkat group
C: LAT mutation group

Figure 3 The histopathological examination of tumor( $\times 400$ times $)$

\section{FCM detection}

Saw the figure for PB tumor cell proliferation. Proportion of CD59 positive cells of LAT high expression group was higher than that of other two groups, $P<0.05$. LAT mutation group was lower than the normal Jurkat cell group, $P$ $<0.05$ showed that the difference had statistical significance (Figure 4).

A

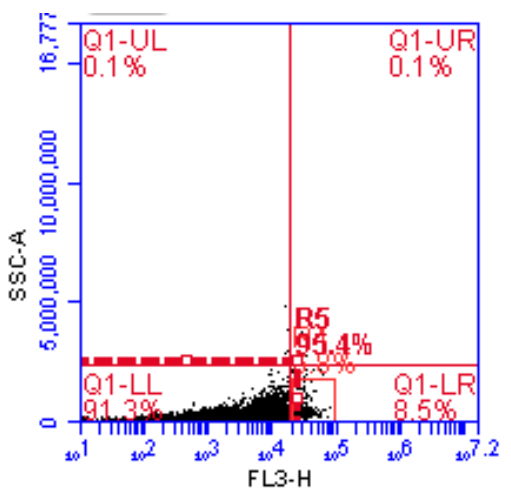

C

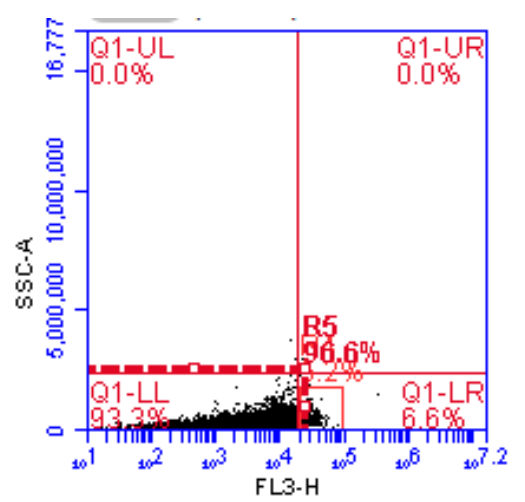

B

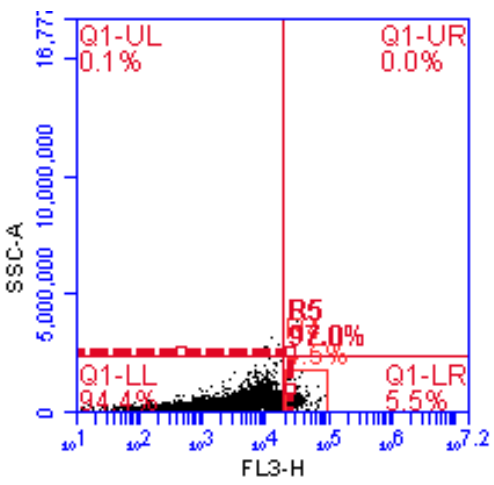




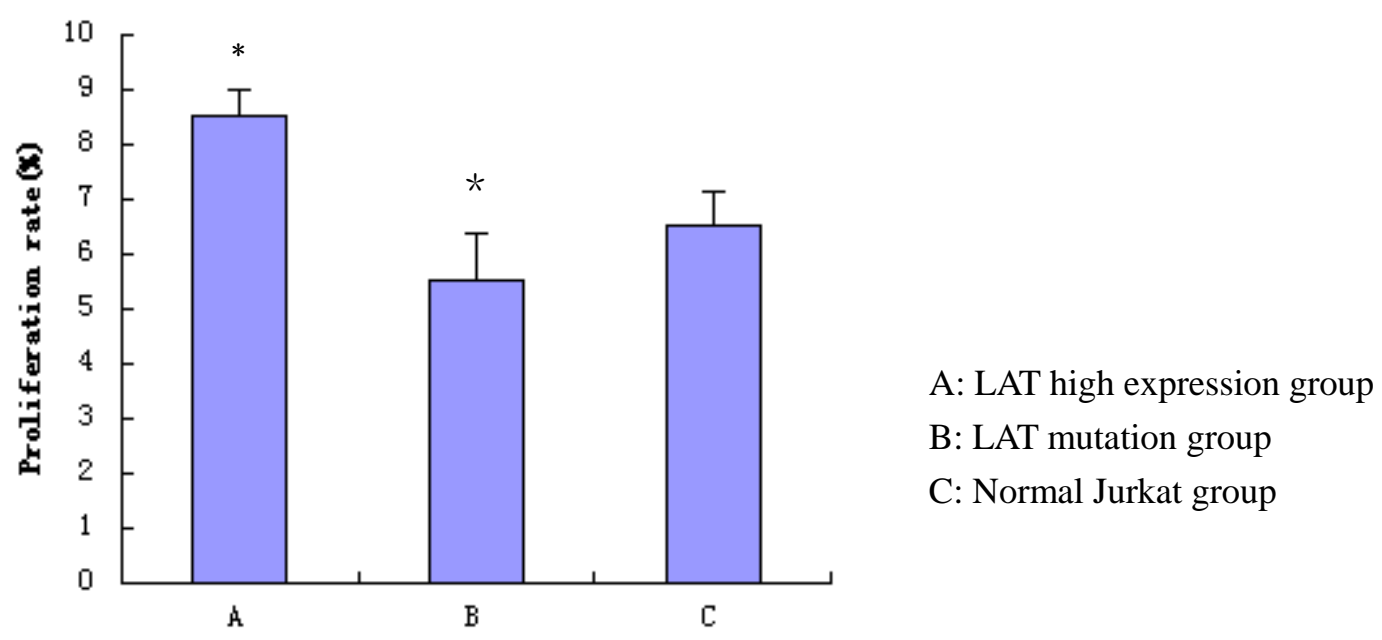

Figure 4 The result of FCM detection

\section{Expression level of IL-2}

In serum, ELISA testing result showed that LAT high expression group has higher IL-2 level than other two groups, $P<0.05$. IL-2 level of LAT mutation group was lower than that of normal Jurkat group, $P<0.05$ showed that the difference had statistical significance. It indicated that $\mathrm{T}$ cell activation level of LAT high expression group was far higher than that of LAT mutation group and normal Jurkat group (Figure 5).

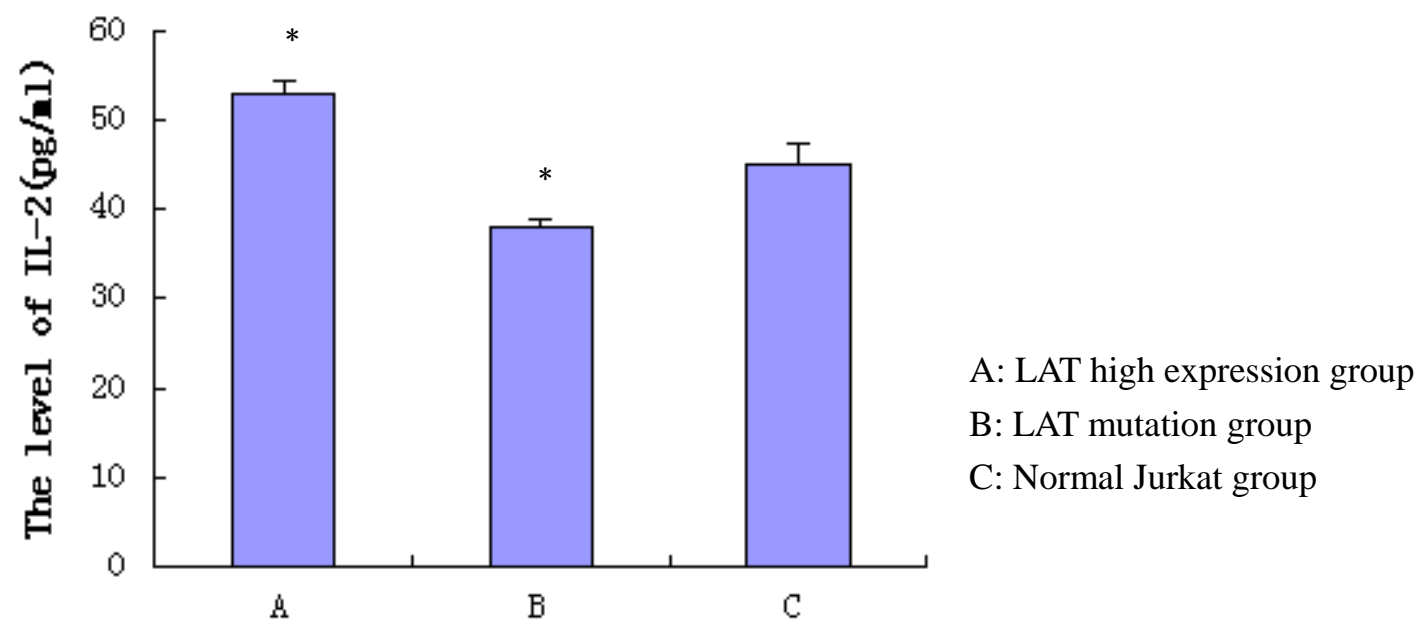

Figure 5 The level of IL-2 in ELISA testing $(* P<0.05)$

\section{Discussion}

LAT is a transmembrane protein that becomes rapidly tyrosine-phosphorylated after TCR engagement ${ }^{[11]}$. As an adaptor protein in $\mathrm{T}$ lymphocyte activation, LAT palmitic acidification is important to the signal transduction process and location on the membrane of plasma cells ${ }^{[12]}$, LAT of non-palmitoylation is easy to be decomposed ${ }^{[13,14]}$. From its structure, we can learn that LAT molecule extracellular domain is short, and the cytoplasm domain is relatively long ${ }^{[2,15]}$. However, the study shows that LAT extracellular section is of great importance to its own stability. Especially, its palmitic acid guarantees LAT locating on GEMs, this microdomain palys an inportant role in signal transduction after TCR engagement ${ }^{[16,17]}$. It is reported that lipid rafts is a general term used to describe microdomains at membrane, and enriched in glycosphingolipids, sphingomyelin and cholesterol creating an environment which attracts certain proteins while excludes others ${ }^{[18-20]}$. 
The study shows that the anchoring structure of glycosyl phosphatidylinositol (GPI) of CD59 molecule ${ }^{[21]}$, a Ly6 superfamily member is the basis of signal transduction, which has palmitic acid group needed by $\mathrm{LAT}^{[22]}$. Our research group has proved that CD59 can be located on lipid raft by providing the acid group for palmitic acidification needed by LAT and further finish signal transduction ${ }^{[23]}$. This research further compares the impacts of LAT high expression and LAT palmitic acidification locus mutation on cell proliferation based on nude mouse tumorigenesis and then analyzes activation effect of LAT molecule in T cell signal transduction.

The research finds that morbidity of 5-week mice is $100 \%{ }^{[24]}$. Therefore, we use 5-week female Balb/c nude mice. After injecting leukemia cells at armpits, there are visible lumps in armpits in the second week. The tumors also get larger than larger. Till the third week, the early symptoms of leukemia appear, which shows that the tumor cells have spread in the mouse body. Considering the small week age of the experimental mice, after CTX pretreatment, the number of tumor cells gets larger, which greatly enhances the success rate of modeling. Pathological HE staining shows that tumor tissue of LAT high expression group is dense and has abundant blood vessels. The research shows that after monoclonal antibody detection, CD59 expresses high in Jurkat cell series $^{[25]}$. Therefore, CD59 positive rate is taken as the indicator of tumor cell invasion. Human tumor cell surpassing $1 \%$ in tumor-bearing mice is taken as the implantation standard ${ }^{[26]}$. FCM result shows that the three groups all have tumor cell proliferation. Proportion of positive cell in PB of LAT high expression group reaches $8.5 \%$. IL-2 is mainly generated by the activated $\mathrm{CD}^{+} \mathrm{T}$ cells and can influences $\mathrm{T}$ cell proliferation ${ }^{[27-29]}$. The level of IL-2 would reduced when the avtivation of $\mathrm{T}$ cells is be interfered $^{[30]}$. In this research IL-2 secretion level of LAT high expression group is higher than other two groups, which is closely related to the activation effect of LAT molecules to $\mathrm{T}$ lymphocyte proliferation.
In this research, a Jurkat cell nude mouse tumor model was established. The identification result shows that LAT can locate to lipid raft through palmitic acidification and can continue transducing signals. After palmitic acidification site mutation, $\mathrm{T}$ cell activation signal transduction will be restrained. This research will provide a new target for $\mathrm{T}$ lymphocytic leukemia gene therapy.

\section{SUPPLEMENTARY MATERIALS}

Foundation: The national natural science foundation of China.(81273206)

Contribution: Experimental design for the corresponding author, the implementation for the first author, auxiliary experiment for the third, four, five. The first author written,the corresponding author reviewed.

Conflict of benefit: It isn't involve.

Requirement of ethics: Handling of the animals is coincide with the rules of related animal ethics standards for the 2009 《Ethical issues in animal experimentation》 in the progress.

Statement: The article is original works, no plagiarism and no leaks, contents and datas are real.

\section{REFERENCE}

1) Xia BS. The relationship on adapter protein and $\mathrm{T}$ cell signal transduction. Molecular Diagnostics. 2009;1: 47-53.

2) Zhang W, Sloan-Lancaster J, Kitchen J, et al. LAT: the ZAP-70 tyrosine kinase substrate that links $\mathrm{T}$ cell receptor to cellular activation. Cell, 1998; 92(1): 83-92.

3) Wang K, Wang $\mathrm{C}, \mathrm{Wu} \mathrm{W}$, et al. Establishment of animal model of human cervical cancer in BALB/c nude mice by tumor tissue xenograft. Zhonghua Lao Dong Wei Sheng Zhi Ye Bing Za Zhi. 2014;32(6):441-3.

4) Malbec O, Malissen M, Isnardi I, et al. Linker for Activation of T Cells Integrates Positive and Negative Signaling in Mast Cells. Immunoal, 2004; 173(8):5086-5094.

5) Ragab A, Severin S, Gratacap MP, et al. Roles of the C-terminal tyrosine residuces of LAT in GPVI-induced platelet activation: insights into the mechanism of PLC gamma 2 activation. Blood, 2007;110(7):2466-2474.

6) Su YW, Jumaa H. LAT links the pre-BCR to calcium 
signaling. Immunity, 2003;19:295-305.

7) Facchetti F, Chan JK, Zhang W, et al. Linker for activation of $\mathrm{T}$ cells (LAT), a novel immunohistochemical marker for $\mathrm{T}$ cells, NK cells, mast cells, and megakaryocytes: evaluation in normal and pathological conditions. AM J Pathol, 1999; 154:1037-1046.

8) Zhang W, Trible RP, Samelson LE. LAT palmitoylation: its essential role in membrane microdomain targeting and tyrosine phosphorylation during T cell activation. Immunity, 1998; 9(2):239-46.

9) QIN Y, GAO MH, et al. Construction and subcellular localization of LAT-EGFP fusion protein[J].China Immunology,2013;(29):526-533.

10) Ponimaskin E, Dityateva G, Ruonala MO, et al. Fibroblast growth factor-regulated palmitoylation of the neural cell adhesion molecule determines neuronal morphogenesis. J Neurosci, 2008; 28:8897-8907.

11) Wange RL. LAT, the linker for activation of T cells: a bridge between $\mathrm{T}$ cell-specific and general signaling pathways.

12) Lin J, Weiss A, Finco TS. Localization of LAT in glycolipid-enriched microdomains is required for $\mathrm{T}$ cell activation. J Biol Chem, 1999; 274:28861-28864.

13) Gringhuis SI, Leow A, Papendrecht-Van Der Voort EA, et al. Displacement of linker for activation of $\mathrm{T}$ cells from the plasma membrane due to redox balance alterations results in hyporesponsiveness of synovial fluid $\mathrm{T}$ lymphocytes in rheumatoid arthritis. Immunol, 2000;164: 2170-2179.

14) Hundt M, Tabata $H$, Jeon MS, et al. Impaired activation and localization of LAT in anergic $\mathrm{T}$ cells as a consequence of a selective palmitoylation defect. Immunity, 2006;24: 513-522.

15) Weber JR, Orstavik S, Torgersen KM, et al. Molecular cloning of the cDNA encoding pp36, a tyrosine-phosphorylated adaptor protein selectively expressed by $\mathrm{T}$ cells and natural killer cells. Exp Med. 1998; 187(7):1157-61.

16) Tanimura N, Saitoh $S$, Kawano $S$, et al. Palmitoylation of LAT contributes to its subcellular localization and stability. Biochem Biophys ResComun. 2006;341(4):1177-1183.

17) Zhang W, Trible RP, Zhu M, et al. Association of Grb2, Gads, and phospholipase C-gamma 1 with phosphorylated LAT tyrosine residues. Effect of LAT tyrosine mutations on $\mathrm{T}$ cell angigen receptor-mediated signaling. J Biol Chem, 2000;275(30):23355-23361.

18) Simons K, Ikonen E. Functional rafts in cell membranes. Nature. 1997;387:569-572.

19) Edidin M. Lipid microdomains in cell surface membranes. Curr Opin Struct Biol. 1997; 7:528-532.

20) Brown DA, London E. Functions of lipid rafts in biological membranes. Annu Rev Cell Dev Biol.1998;14:111-136.

21) Kimberley FC, Sivasankar B, Paul Morgan B. Alternative roles for CD59. Mol Immunol, 2007;44:73-81.

22) Meri S, Morqan BP, Davies A, et al. Human protectin (CD59), an 18,000-20,000 MW complement lysis restricting factor, inhibits C5b-8 catalysed insertion of C9 into lipid bilayers [J]. Immunology,1990;71:1-9.

23) Gao MH, Li YY, Wang LN, et al. CD59 has a positive effect on LAT induced T lymphocytes activation. Chinese Journal of Immunology, 2014;(7)

24) Ohsugi $T$, Yamaguchi $K$, Kumasaka $T$, et al. Rapid tumor death model for evaluation of new therapeutic agents for adult T-cell leukemia[J]. Lab Invest, 2004;84(2): 263-266.

25) Bai Y, ZHU XH. Preparation and identifacation of anti-CD59 monoclonal antibody. Immunological Journal, 1994; 10(5):218.

26) Wang JC, Lapidot $\mathrm{T}$, Cashman JD, et al. High level engraftment of NOD/SCID mice by primitive normal and leukemic hematopoietic cells from patients with chronic myeloid leukemia in chronic phase[J].Blood,1998;91(7):2406-14.

27) Miyazaki T, Liu ZJ, Kawahara A, et al. Three distinct IL-2 signaling pathways mediated by bcl-2, cmyc, and lck cooperate in hematopoietic cell proliferation. Cell, 1995; 81(2):223-31.

28) Morgan DA, Ruscetti FW, Gallo R, et al. Selective in vitro growth of $\mathrm{T}$ lymphocytes from normal human bone marrows. Science, 1976; 193(4257):1007-1008.

29) Lenardo MJ. Interleukin-2 programs mouse alpha beta $T$ lymphocytes for apoptosis. Nature, 1991; 353(6347): 858-61.

30) Donlin L, Roman CA, Adlam M, et al. Defective thymocyte mat uration by transgenic expression of a truncated form of the T lymphocyte adapter molecule and Fyn substrate, Sin. Immunol, 2002; 169(12):6900-9. 